Check for updates

Cite this: RSC Adv., 2020, 10, 28644

\section{Synthesis of benzonaphthofuroquinones and benzoylnaphthindolizinediones by reactions of flavonoids with dichlone under basylous, oxygenous and aqueous conditions: their cytotoxic and apoptotic activities $\uparrow$}

\author{
Peng Luo, ${ }^{\text {ac }}$ Wanxing Wei, ${ }^{\text {a }}$ Saqlain Haider, ${ }^{\text {b }}$ Shabana I. Khan, ${ }^{\mathrm{b}}$ Mei Wang, ${ }^{\mathrm{b}}$ \\ Weigao Pan (D)*ac and Amar G. Chittiboyina*b
}

\begin{abstract}
Using flavonoids and dichlone as substrates, benzonaphthofuroquinones (1, 2, 3, 5, 6, novel; 4 new) and benzoylnaphthindolizinediones (7, 8, known; 9 , new) were synthesized through common base-catalyzed method and a new method of combining base-catalyzed with $\mathrm{O}_{2} / \mathrm{H}_{2} \mathrm{O}$ exposing. The possible reaction mechanisms may involve the process like isomerization, hydration, oxidation, decomposition and intermolecular condensation. Benzonaphthofuroquinones $(2,3,4,5)$ were found to exhibit potent cytotoxicity against carcinoma cell lines and low toxicity to normal cell lines. The compounds 4 and 5 not only expressed a significant late-stage-apoptosis against human leukemia and melanoma, but also promoted the cleavage of caspase- 3 and PARP in human leukemia, which suggested that the late-stageapoptosis and caspase-3 pathway may be responsible for the cytotoxicities of these benzonaphthofuroquinones. The replacement of the furan ring with pyrrole system in benzoylnaphthindolizinediones $(7,8,9)$ resulted in the loss of anticancer activity.
\end{abstract}

Received 10th July 2020 Accepted 29th July 2020 DOI: $10.1039 / \mathrm{d}$ Ora06043g rsc.li/rsc-advances mechanism give poor selectivity for cytotoxicities between carcinoma and normal cells, and its furan ring attached to the naphthoquinone scaffold along with the lipophilic character of the alkyl substituents is a key feature required for the enhancement of anticancer activity. ${ }^{4}$ While, dinaphtho[1,2$\left.b: 2^{\prime}, 3^{\prime}-d\right]$ furan-7,12-dione derivatives (Scheme 1, A5), with additional naphtha-moiety on $\alpha$-dunnione, had anticancer mechanisms of topoisomerase-I mediated DNA cleavage, epidermal and vascular endothelial growth factor receptors inhibitory actions, which mechanisms owned high selectivity for cytotoxicities. ${ }^{5}$

So modifying dunniones with aromatic ring-group may be a good way to achieve anticancer mechanisms correlated with cancer-related-biomacromolecules, which may result in high selectivity for cytotoxicity. Flavonoids were potential phenol source for modifying uses, in which the two phenol moieties and a variable central-three-carbons-chain may provide various substituent groups. Meanwhile, constructing other phenolmodified $\alpha$-dunnione-like derivatives (such as using pyrrol system instead of furan system) may be useful to investigate the changes of cytotoxicities or anticancer mechanisms.

In reported methods, benzo- or naphthanaphthofuroquinones ( $\alpha$-dunniones analogs) was synthesized mainly through base-catalyzed condensation reaction. Condensation of dichlone with methyl 1,4-dihydroxy-2-naphthoate or 2-acetyl1,4-dihydronaphthalene with presence of $\mathrm{K}_{2} \mathrm{CO}_{3}$ under reflux 
<smiles>CC1OC2c3ccccc3C(=O)C(=O)C2C1(C)C</smiles>

A1<smiles>CC1OC2=C(C(=O)c3ccc(O)cc3C2=O)C1(C)C</smiles>

A2<smiles>CC1OC2=C(C(=O)c3ccccc3C2=O)C1(C)C</smiles>

A3<smiles>C=C(C)C1CC2=C(O1)C(=O)c1cccc(O)c1C2=O</smiles>

A4<smiles></smiles>

A5

Scheme 1 The reported dunnione analogs with cytotoxicity.

in pyridine at $90{ }^{\circ} \mathrm{C}$ resulted in dinaphtho[1,2-b:20,30- $\left.d\right]$ furan7,12-dione (Scheme 2, B1) ${ }^{6}$ or 6-acetyl-5-hydroxydinaphtho[2,3$\left.b: 2^{\prime}, 1^{\prime}-d\right]$ furan-7,12-dione (Scheme 2, B2), ${ }^{7}$ respectively. [1,3] Dioxolo[4,5-f]naphtho[2,3-b] benzofuran-6,11-dione (Scheme 2, B3) or 1,3-dihydroxybenzo[b]naphtho[2,3-d]furan-6,11-dione (Scheme 2, B4) was synthesized from the reaction of dichlone with sesamol (or phloroglucinol) in presence of $\mathrm{KOH}$ under reflux in pyridine at $90^{\circ} \mathrm{C}\left(\right.$ or $\left.30^{\circ} \mathrm{C}\right) .{ }^{8}$

Intermolecular C-C-bond formation and intermolecular $O$ alkylation were the two condensation processes for the synthesis of naphthofuroquinones scaffold. Other methods of preparation included light irradiation and oxidation were reported. 2-Hydroxynaphtho-1,4-dione was converted to negative ion by pyrex-filtered light, and reacted with 2-naphthol exciplex to produce a $\mathrm{C}-\mathrm{C}$ bond firstly, and then to form a C-O bond by dehydration. ${ }^{9}$ Quinone-arenols were prepared from hydroquinone monomethylethers by oxidants to form $\mathrm{C}-\mathrm{C}$ bond in the first step, and by oxidative cyclization of quinone-arenols to give $\mathrm{C}-\mathrm{O}$ bond in the second step to produce dibenzofuranquinones. ${ }^{10}$

In reported methods, benzoylnaphthindolizinediones $(\alpha$ dunnione-like compounds) were synthesized by potassium carbonate-catalyzed one-pot tandem reactions of the $\mathrm{N}$-ylides with 2,3-dichloro-1,4-naphthoquinone (Scheme 3, (1)), ${ }^{11}$ or by copper(II)-catalyzed three-component reactions of 2-bromoacetophenone, 1,4-naphthoquinone, and pyridine via $\mathrm{sp}^{2}-\mathrm{C}-\mathrm{H}$ difunctionalization of naphthoquinone followed by intramolecular cyclization and oxidative aromatization (Scheme 3, (2)), ${ }^{12}$ or by iodine oxidation of 2-alkyl-1,4-naphthoquinones in the presence of substituted pyridines (Scheme 3, (3)). ${ }^{13}$

In our initial plan, in order to get naphthanaphthofuroquinones, dihydroflavone and chalcone were respectively used to react with dichlone in pyridine under anoxic and anhydrous conditions, however, no targeted products were obtained. Unintentionally, the anoxic and anhydrous conditions being removed and reaction system being exposed to the moist air, unexpected benzoylnaphthindolizinediones were found. So this $\mathrm{O}_{2} / \mathrm{H}_{2} \mathrm{O}$ exposing combining with base-catalyzed method were developed.

With regard to recent works about the utilization of water to promote reaction, ecofriendly and efficient synthesis of sulfonylated $N$-heteroaromatics ${ }^{14}$ and 2 -sulfonyl quinolines/pyridines ${ }^{15}$ were conducted in water, and in the iodine-catalyzed odorless synthesis of $S$-thiocarbamates, water played a dual role, viz. co-solvent and $\mathrm{H} / \mathrm{O}$ source. ${ }^{16}$<smiles>[R]OC(=O)c1cc(O)c2ccccc2c1O</smiles>

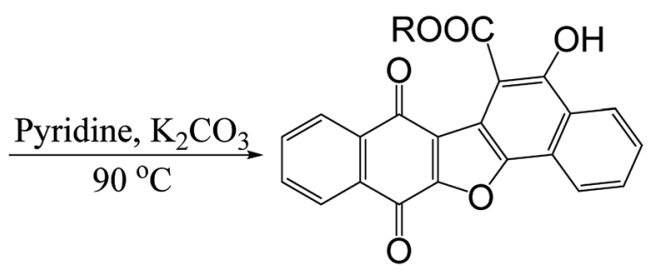

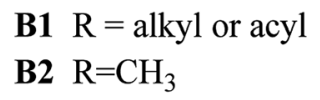<smiles>[R]c1cc(O)cc(O)c1[R2]</smiles><smiles>O=C1C(Cl)=C(Cl)C(=O)c2ccccc21</smiles><smiles>[R5]c1cc2oc3c(c2c([R])c1[R])C(=O)c1ccc(C#CC(=O)O)cc1C3=O</smiles>

B3 $\mathrm{R}_{3}=\mathrm{H}, \mathrm{R}_{4}+\mathrm{R}_{5}=\mathrm{OCH}_{2} \mathrm{O}$

B4 $\mathrm{R}_{3}=\mathrm{OH}, \mathrm{R}_{4}=\mathrm{H}, \mathrm{R}_{5}=\mathrm{OH}$

Scheme 2 The reported synthetic methods for benzo- or naphthanaphthofuroquinones. 


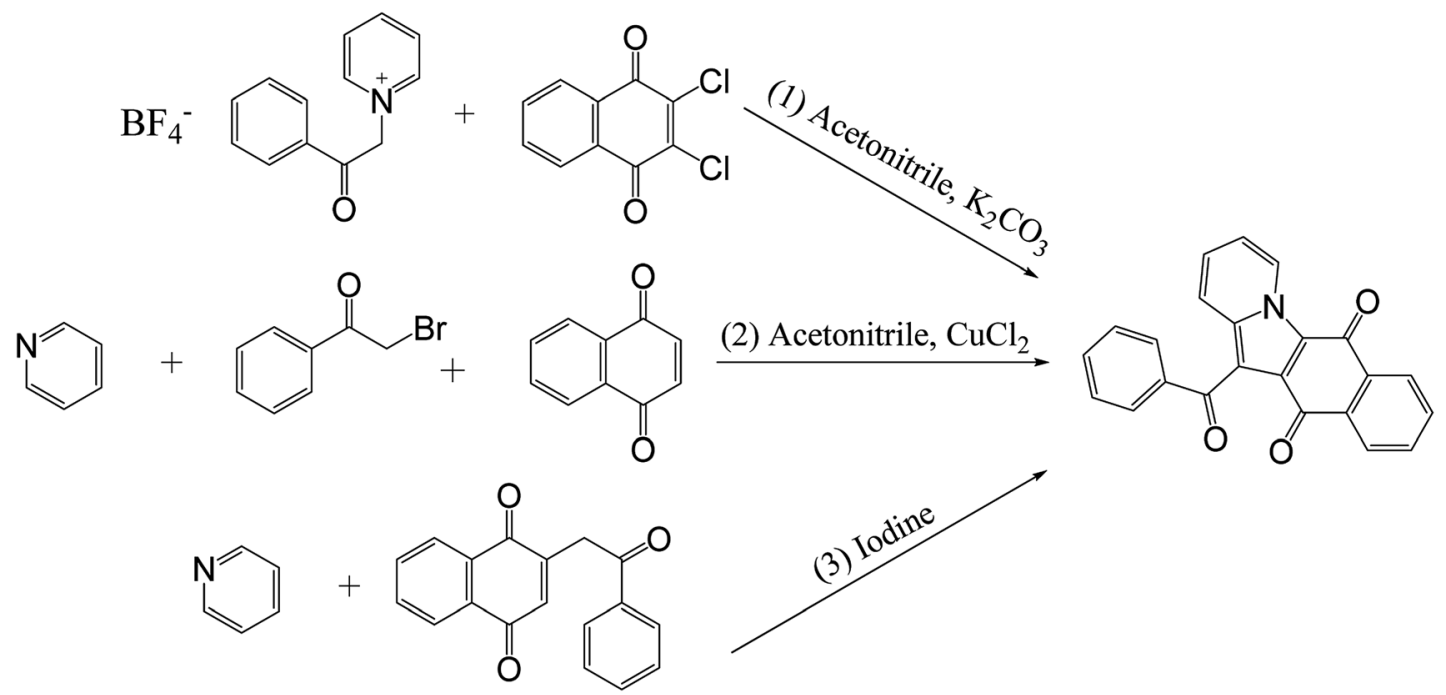

Scheme 3 The reported synthetic methods for benzoylnaphthindolizinediones.

\section{Results and discussion}

\subsection{Chemistry}

2.1.1 Structure elucidation and characterization. Ten derivatives were synthesized here, viz. 3,4-dihydroxy-1-(3,5,7trihydroxy-4-oxo-4H-chromen-2-yl)naphtho[2,3-b]benzofuran6,11-dione (1), 4-hydroxy-3-((3-hydroxy-1,4-dioxo-1,4-dihydronaphthalen-2-yl)oxy)-1-(3,5,7-trihydroxy-4-oxo-4H-chromen2-yl)naphtho[2,3-b]benzofuran-6,11-dione (2), 3-hydroxy-4-((3hydroxy-1,4-dioxo-1,4-dihydronaphthalen-2-yl)oxy)-1-(3,5,7trihydroxy-4-oxo-4H-chromen-2-yl)naphtho[2,3-b]benzofuran6,11-dione (3), 4-hydroxynaphtho[2,3-b]benzofuran-6,11-dione (4), 4-hydroxy-1-(3,5,7-trihydroxy-4-oxo-4H-chromen-2-yl)naphtho[2,3- $b]$ benzofuran-6,11-dione (5), 4-((3-hydroxy-1,4-dioxo-1,4dihydronaphthalen-2-yl)oxy)-1-(3,5,7-trihydroxy-4-oxo-4H-chromen-2-yl)naphtho[2,3- $b]$ benzofuran-6,11-dione (6), 12-(2hydroxybenzoyl)benzo[f]pyrido[1,2- $a$ ]indole-6,11-dione (7), 12benzoylbenzo[f]pyrido[1,2- $a]$ indole-6,11-dione (8), 12-(4hydroxybenzoyl)benzo[f]pyrido[1,2-a]indole-6,11-dione (9), 1,4dioxo-3-(pyridin-1-ium-1-yl)-1,4-dihydronaphthalen-2-olate (10, side product).

Structures of 1-10 were elucidated and characterized by NMR $\left({ }^{1} \mathrm{H},{ }^{13} \mathrm{C}\right.$, DEPT,${ }^{1} \mathrm{H}-{ }^{1} \mathrm{H}$ COSY, HSQC, HMBC), MS (ESI-MS, APCI-MS and HR ESI-MS) and IR spectra (see the ESI $\dagger$ file).

In NMR spectra of $\mathbf{1}$, total of seven aromatic methines signals were observed. As concerns resonances changes occurring on the B-ring of flavonoid moiety, one methane signal at $\delta_{\mathrm{H}} / \delta_{\mathrm{C}} 7.36(\mathrm{~s}) / 118.6$ (position 15) was remained while the other one disappeared, which suggested that intermolecular $\mathrm{C}-\mathrm{C}$ condensation reaction occurred in position 11. Furthermore, a new aromatic oxygenated carbon signal at $\delta_{\mathrm{C}} 154.1$ (C-2) was found, so an additional $\mathrm{C}-\mathrm{O}$ covalent bond was formed, which contributed to produce the furan ring (Scheme 4). As compared with $\mathrm{H}-15 / \mathrm{C}-15$ resonances of 1 at $\delta_{\mathrm{H}-15} / \delta_{\mathrm{C}-15} 7.36 / 118.6$, the corresponding resonances of 2 moved to up-field shift at $\delta_{\mathrm{H}-15} /$ $\delta_{\mathrm{C}-15} 7.29 / 113.3$, and as to $3, \delta_{\mathrm{H}-15}$ move to down-field shift at
7.56 while $\delta_{\mathrm{C}-15}$ almost unchanged at 117.8 , which meaned that the second dichlone moiety, through intermolecular $O$-alkylation condensation, was located at $14-\mathrm{O}$ (ortho effect) in 2 , and at $13-\mathrm{O}$ in 3 (Scheme 4).

In case of 5, eight aromatic methines signals were found. As for resonances changes occurring on the B-ring of flavonoid moiety, two methine-protons signals at $\delta_{\mathrm{H}} 7.22-7.24(1 \mathrm{H}, \mathrm{d}, J=$ $8.3 \mathrm{~Hz})]$ and $\delta_{\mathrm{H}} 7.61-7.63(1 \mathrm{H}, \mathrm{d}, J=8.3 \mathrm{~Hz})$ with their coupling in ${ }^{1} \mathrm{H}-{ }^{1} \mathrm{H}$ COSY were observed while the third one vanished, which meant that $\mathrm{C}-\mathrm{C}$ condensation reaction occurred in position 11 (Scheme 4). Furthermore, a new aromatic oxygenated carbon signal at $\delta_{\mathrm{C}} 154.7$ (C-2) was found, so an additional $\mathrm{C}-\mathrm{O}$ covalent bond was formed to produce the furan ring (Scheme 4). 6 manifested obvious proton/carbon resonances changes on the B-ring comparing to those of $\mathbf{5}$, so the second naphthoquinone moiety was located at $13-\mathrm{O}$ (Scheme 4). 4 was produced from 5 (Scheme 4), so the hydroxyl group was located at position 13, which had been confirmed by its NMR data and single crystal structure in our previous work. ${ }^{17}$

7 had been confirmed by its NMR data and single crystal structure in our previous work. ${ }^{18}$

By the coupling relationships in ${ }^{1} \mathrm{H}-{ }^{1} \mathrm{H}$ COSY of 8 , proton signals of $\delta_{\mathrm{H}} 7.91-7.93(2 \mathrm{H}, \mathrm{d}, J=7.5 \mathrm{~Hz}), 7.46-7.49(2 \mathrm{H}, \mathrm{t}, J=$ $7.5 \mathrm{~Hz}), 7.60-7.63(1 \mathrm{H}, \mathrm{t}, J=7.5 \mathrm{~Hz})$ were assigned to a fiveconsecutive-methines-chain of phenyl group. Considering the facts that the HR ESI-MS spectra gave a molecular formula of $\mathrm{C}_{23} \mathrm{H}_{13} \mathrm{NO}_{3}$ and pyridine was used as solvent in the reaction, proton signals of $\delta_{\mathrm{H}} 9.83-9.85(1 \mathrm{H}, \mathrm{d}, J=7.0 \mathrm{~Hz}), 7.21-7.24(1 \mathrm{H}$, $\mathrm{t}, J=7.3 \mathrm{~Hz}), 7.41-7.44(1 \mathrm{H}, \mathrm{m}), 7.97-7.99(1 \mathrm{H}, \mathrm{d}, J=9.0 \mathrm{~Hz})$ were assigned to a four-consecutive-methines-chain of pyridine moiety by the ${ }^{1} \mathrm{H}-{ }^{1} \mathrm{H}$ COSY coupling relationships, which meant that pyridine was involved in the intermolecular condensation reaction (Scheme 5).

Instead of five consecutive coupling protons in $\mathbf{8}$, four protons with coupling relationships between signals of $\delta_{\mathrm{H}} 6.80-$ $6.82(2 \mathrm{H}, \mathrm{d}, J=8.5 \mathrm{~Hz})$ and those of $\delta_{\mathrm{H}} 7.73-7.77(2 \mathrm{H}, \mathrm{m})$ were 


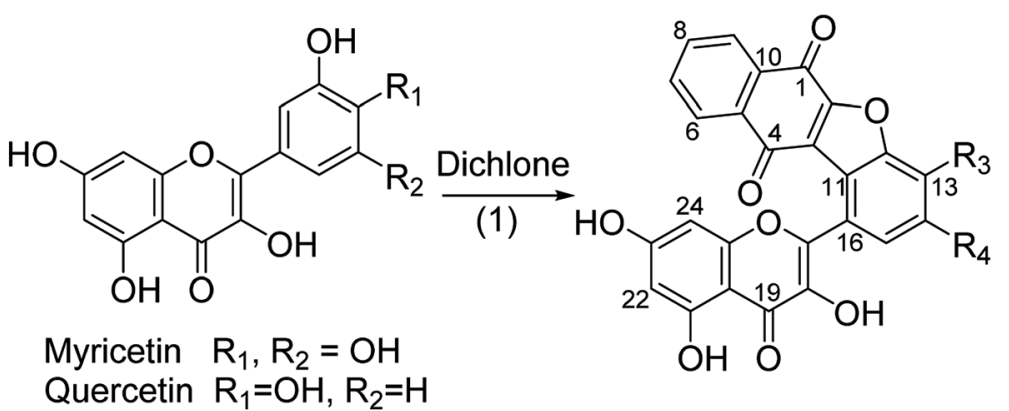<smiles>COC1=C(O)C(=O)c2ccccc2C1=O</smiles>
$\begin{array}{ll}\text { Myricetin } & \mathrm{R}_{1}, \mathrm{R}_{2}=\mathrm{OH} \\ \text { Quercetin } \mathrm{R}_{1}=\mathrm{OH}, \mathrm{R}_{2}=\mathrm{H}\end{array}$

$1 \mathrm{R}_{3}, \mathrm{R}_{4}=\mathrm{OH}$

$2 \mathrm{R}_{3}=\mathrm{OH}, \mathrm{R}_{4}=\mathrm{Q}$

(Derived from myricetin)

$3 \quad \mathrm{R}_{3}=\mathrm{Q}, \quad \mathrm{R}_{4}=\mathrm{OH}$ (Derived from myricetin)
$5 \quad \mathrm{R}_{3}=\mathrm{OH}, \mathrm{R}_{4}=\mathrm{H}$

$6 \quad \mathrm{R}_{3}=\mathrm{Q}, \quad \mathrm{R}_{4}=\mathrm{H}$
(Derived from quercetin)
(Derived from quercetin)

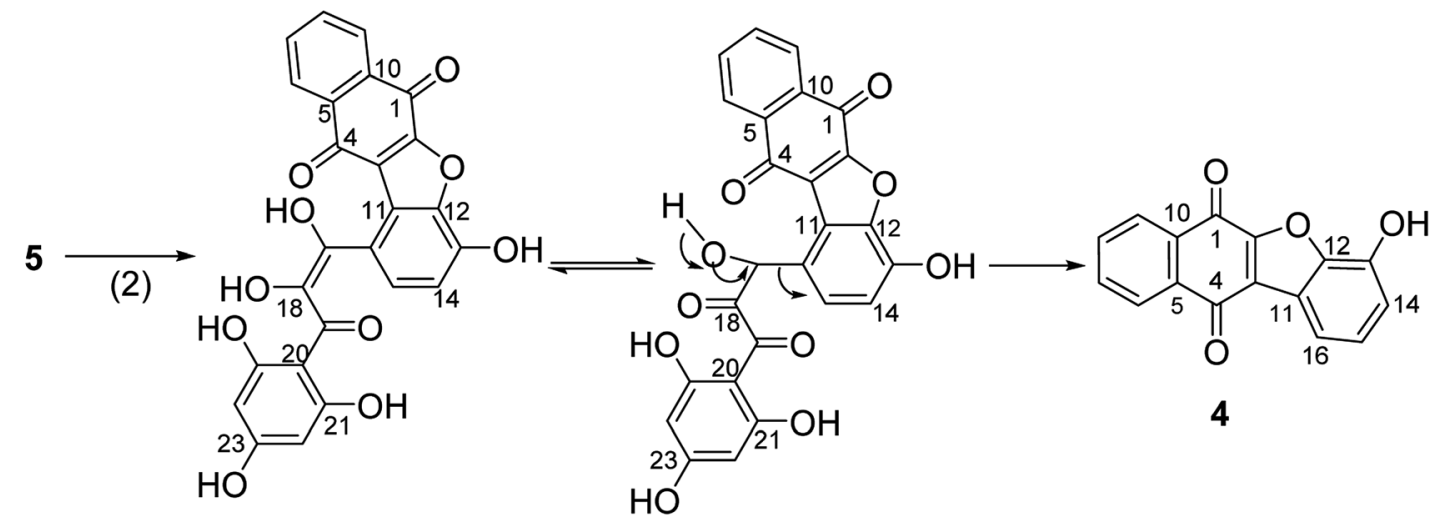

Scheme 4 Synthesis of benzonaphthofuroquinones and the possible reaction mechanism of 4 . Reagents and conditions: $(1)$ pyridine, $85^{\circ} \mathrm{C}$, $24 \mathrm{~h}$; (2) pyridine, moist air $\left(\mathrm{O}_{2} / \mathrm{H}_{2} \mathrm{O}\right)$ injection, $85^{\circ} \mathrm{C}, 24 \mathrm{~h}$.

observed in 9. Furthermore, one more aromatic oxygenated carbon at $\delta_{\mathrm{C}} 162.8$ was found in 9 than in 8 (Scheme 5).

2.1.2 Synthesis and discussion for reaction mechanisms. The preparations of $\mathbf{1}, 2,3,5,6$ were conducted by common methods ${ }^{6,7}$ using flavonols (myricetin or quercetin) as substrates under pyridine-catalyzed conditions (Scheme 4). Besides mono condensation products $(\mathbf{1}, \mathbf{5})$, additional dichlone molecule was introduced to form bis-naphthoquinones compounds $(2,3,6)$ merely by intermolecular $\mathrm{C}-\mathrm{O}$ condensation (Scheme 4).

The structure of $\mathbf{4}$ was confirmed by both single crystal X-ray diffraction and NMR-spectrum in our previous work. ${ }^{17}$ In the condensation reaction of quercetin with dichlone under basylous, anoxic and anhydrous conditions, $\mathbf{4}$ was not found, while providing additional condition of $\mathrm{O}_{2} / \mathrm{H}_{2} \mathrm{O}$ exposing, 4 was obtained (Scheme 4). It may indicate that C-ring of flavonol moiety in 5 was cleaved by hydration, and then a "2,3-dioxo-3-(2,4,6trihydroxyphenyl)propanal" group was eliminated to give a benzonaphthofuroquinone core $(\mathbf{4}$, Scheme 4$)$. However, this "2,3-dioxo-3-propanal" elimination mechanism was not found in the literature, so further work should be done to confirm it.

In Scheme 5, occurrence of side product (10) provided evidence for the existence of intermediate of dichlone reacted with pyridine, and the structure of $\mathbf{1 0}$ and its synthetic method (under similar conditions and using the same substrates) were reported by Andreas Schmidt et al. ${ }^{19}$ Dihydroflavone (naringenin) isomerized into trans-chalcone. Acetophenone blocks, which were generated from trans-chalcone via retro-aldol decomposition after hydration and isomerization, reacted with above intermediate to build a benzoylnaphthindolizinedione skeleton $(7,8,9$, Scheme 5$)$. The fact that 7 and 8 were derived from the different part of chalcone provided evidence for the isomerization reaction of chalcone hydrate.

Two phases of the hydration and dealdolization reactions of chalcone (1,3-diphenyl-2-propen-1-one) and their reaction rate constants were reported by J. Peter Guthrie et al. Hydration of chalcone was catalyzed by aqueous sodium hydroxide to give the hydrate (1,3-diphenyl-3-hydroxy-1-propanone) firstly, and then retro-aldol reaction of this hydrate was also catalyzed by aqueous sodium hydroxide to produce benzaldehyde and acetophenone. ${ }^{20}$

\subsection{Biology}

2.2.1 Cytotoxicity. Some of the benzonaphthofuroquinones $(1,2,3,4,5)$ exhibited anticancer effects, while the benzoylnaphthindolizinediones $(7,8,9)$ showed no activities (Table 1 ). The formed furan-ring in benzonaphthofuroquinones being replaced by pyrrol-ring in benzoylnaphthindolizinediones resulted in the loss of anticancer activity (Table 1).

Doxorubicin manifested strong cytotoxicities against both carcinoma and normal cell lines, while 1, 2, 3, 4, 5 possessed good anticancer activities and low toxicity to normal cells. 3 owed high antagonistic effect against almost all test solid carcinoma cell lines, while 2, 5 just showed good inhibition against SK-MEL (solid carcinoma), and $\mathbf{4}, \mathbf{5}$ had good inhibition 
<smiles>O=C(/C=C/c1ccccc1)c1ccccc1O</smiles><smiles></smiles><smiles>C=CC(=C)C(O)CC(=O)c1ccccc1O</smiles><smiles>C/C=C\CCCCCO</smiles>

2'-Hydroxychalcone<smiles>C=C1CCCCC1</smiles>

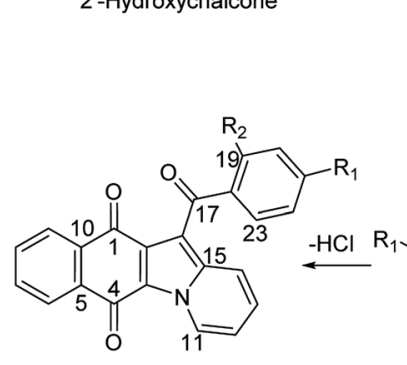<smiles>[R7]c1ccc(C(=O)C(CC)C2C=CC=CN2C2=C(Cl)C(=O)c3cc(I)ccc3C2=O)c([R2])c1</smiles>

(1) $7 \quad \mathrm{R}_{1}=\mathrm{H}, \quad \mathrm{R}_{2}=\mathrm{OH}$ (Derived from 2'-Hydroxychalone)

(2) $8 \quad \mathrm{R}_{1}=\mathrm{H}, \quad \mathrm{R}_{2}=\mathrm{H}$ (Derived from 2'-Hydroxychalone)

(3) $9 \mathrm{R}_{1}=\mathrm{OH}, \mathrm{R}_{2}=\mathrm{H}$ (Derived from Naringenin)<smiles>CC(C)(C)CC(=O)c1c(O)cc(O)cc1O</smiles><smiles>O=C1C([O-])=C([n+]2ccccc2)C(=O)c2ccccc21</smiles>

Scheme 5 Synthesis of benzoylnaphthindolizinediones and the possible reaction mechanisms. Reagents and conditions: pyridine, moist air ( $\mathrm{O}_{2} /$ $\mathrm{H}_{2} \mathrm{O}$ ) injection, $85^{\circ} \mathrm{C}, 24 \mathrm{~h}$.

against HL-60 (non-solid carcinoma) (Table 1). The potent cytotoxic derivatives $(\mathbf{2}, \mathbf{3}, \mathbf{4}, \mathbf{5})$ were selected for further apoptotic test and western blotting analysis.

2.2.2 Apoptosis. In the case of apoptosis against human leukemia (Fig. S1, $\dagger$ see the ESI $\dagger), 2(20 \mu \mathrm{M})$ showed no apoptotic activity; and $3(20 \mu \mathrm{M})$ manifested some slight late-stageapoptosis (Q2, Annexin $\mathrm{V}^{+} / 7-\mathrm{AAD}^{+}$) and early-stage-apoptosis (Q3, Annexin $\left.\mathrm{V}^{+} / 7-\mathrm{AAD}^{-}\right)$; while 4, 5 significantly induced Annexin V-positive apoptosis (with apoptotic proportions of
$53.7 \%$ and $27.9 \%$, respectively) at low concentration ( $5 \mu \mathrm{M})$, and the apoptotic proportions mainly distributed in the upper right quadrant (Annexin $\mathrm{V}^{+} / 7-\mathrm{AAD}^{+}$, late-apoptotic-stage) with proportions of $36.8 \%$ and $20.1 \%$, respectively.

With regard to apoptosis against melanoma (Fig. S2, $\uparrow$ see the ESI $\dagger), 2(30 \mu \mathrm{M})$ and $3(30 \mu \mathrm{M})$ had no apoptotic activities; while $4(30 \mu \mathrm{M})$ and $5(30 \mu \mathrm{M})$ induced obvious Annexin V-positive apoptosis (45.8\% and $17.0 \%$, respectively), in which, the major apoptotic proportions (41.7\% and $15.1 \%$, respectively)

Table 1 Cytotoxicities against carcinoma and normal cell lines

\begin{tabular}{|c|c|c|c|c|c|c|c|}
\hline \multirow[b]{2}{*}{ Treatments } & \multicolumn{5}{|c|}{ Cancer cell lines ${ }^{c}\left(\mathrm{IC}_{50}, \mu \mathrm{M}\right)$} & \multicolumn{2}{|c|}{$\begin{array}{l}\text { Non-cancer cell lines } \\
\left(\mathrm{IC}_{50}, \mu \mathrm{M}\right)\end{array}$} \\
\hline & SK-MEL & KB & BT-549 & SK-OV-3 & HL-60 & LLC-PK1 & Vero \\
\hline 1 & 14.83 & 29.66 & 23.31 & $\mathrm{NA}^{a}$ & $\mathrm{NA}^{a}$ & $\mathrm{NC}^{a}$ & $\mathrm{NC}^{a}$ \\
\hline 2 & 3.88 & 10.87 & 10.87 & 27.95 & 18.17 & 24.84 & 38.82 \\
\hline 3 & 2.02 & 5.90 & 3.57 & 6.21 & 17.32 & 27.95 & 15.53 \\
\hline 4 & 12.88 & 37.88 & 37.88 & 22.73 & 2.57 & 18.18 & 15.91 \\
\hline 5 & 5.26 & 10.96 & 28.51 & 21.93 & 4.29 & 21.93 & $\mathrm{NC}^{a}$ \\
\hline 6 & 39.8 & $\mathrm{NA}^{a}$ & $\mathrm{NA}^{a}$ & $\mathrm{NA}^{a}$ & $\mathrm{NA}^{a}$ & 39.8 & $\mathrm{NC}^{a}$ \\
\hline 7 & $\mathrm{NA}^{a}$ & $\mathrm{NA}^{a}$ & $\mathrm{NA}^{a}$ & $\mathrm{NA}^{a}$ & $\mathrm{NA}^{a}$ & $\mathrm{NC}^{a}$ & $\mathrm{NC}^{a}$ \\
\hline 8 & $\mathrm{NA}^{a}$ & $\mathrm{NA}^{a}$ & $\mathrm{NA}^{a}$ & $\mathrm{NA}^{a}$ & $\mathrm{NA}^{a}$ & $\mathrm{NC}^{a}$ & $\mathrm{NC}^{a}$ \\
\hline 9 & $\mathrm{NA}^{a}$ & $\mathrm{NA}^{a}$ & $\mathrm{NA}^{a}$ & $\mathrm{NA}^{a}$ & $\mathrm{NA}^{a}$ & $\mathrm{NC}^{a}$ & $\mathrm{NC}^{a}$ \\
\hline Doxorubicin & 1.29 & 0.92 & 1.84 & 4.05 & $-^{b}$ & 0.92 & 9.21 \\
\hline Cisplatin & $-{ }^{b}$ & $-{ }^{b}$ & $-{ }^{b}$ & $-{ }^{b}$ & 1.46 & $-{ }^{b}$ & $-{ }^{b}$ \\
\hline
\end{tabular}

${ }^{a}$ NA or NC mean not active up to $40 \mu \mathrm{M} .{ }^{b}$ Not tested. ${ }^{c}$ SK-MEL: malignant melanoma, KB: oral epidermal carcinoma, BT-549: ductal breast carcinoma, SK-OV-3: ovary carcinoma, HL-60: human leukemia. ${ }^{d}$ LLC-PK1: kidney epithelial, VERO: African green monkey kidney fibroblast. 
were observed in the upper right quadrant (Annexin $\mathrm{V}^{+} / 7-\mathrm{AAD}^{+}$, late-apoptotic-stage).

2.2.3 Western blotting analysis. As to HL-60 (Fig. S3-A, $\dagger$ see the ESI $\dagger), 3(20 \mu \mathrm{M}), 4(3 \mu \mathrm{M})$ and $\mathbf{5}(5 \mu \mathrm{M})$ significantly induced the cleavage of caspase- 3 and PARP, while $2(20 \mu \mathrm{M})$ induced obvious PARP-cleavage and slight caspase-3 cleavage, which suggested that $\mathbf{4}$ and $\mathbf{5}$ own relatively higher significant apoptosis inducing effect. With regard to melanoma (Fig. S3-B, $\uparrow$ see the ESI $\dagger$ ), 4, 5 manifested slight apoptosis inducing effect at concentration of $30 \mu \mathrm{M}$.

\section{Experimental}

\subsection{General information and characterization data}

Flash column chromatographic separations were accomplished on Biotage Isolera Four system (Biotage, Uppsala, Sweden). NMR was recorded on DRX-400 and DRX-500 NMR spectrometers (Bruker, Rheinstetten, Germany). ESI-, APCI- and HR ESIMS were recorded on Agilent 1290 LC-MS or Agilent G6530 TOF MS spectrometer (Agilent Technologies Inc., California, USA). Western blot results were visualized on Mini-HD9-Auto Biomolecular imager (Leader Oriental Technology. LTD, Beijing, China). Dichlone and flavonoids were purchased from Fisher Scientific (Fair Lawn, NJ, USA) and Ark Pharm, Inc. (Arlington Heights, IL, USA). Cell lines were gotten from the American Type Culture Collection (Manassas, VA). Caspase-3 (3G2) mouse mAb, caspase-3 antibody, cleaved caspase-3 (Asp175) (5A1E) rabbit mAb, PARP (46D11) rabbit mAb and horseradish peroxidase (HRP) were purchased from Cell Signaling Technology, Inc (Danvers, MA, USA).

3.1.1 Characterization data. 1: orange powder; $315 \mathrm{mg}$, yield $45 \%$ (final product/myricetin $\times 100 \%$ ); dp (decomposition point) $385^{\circ} \mathrm{C}$. ESI-MS: $473.0[\mathrm{M}+\mathrm{H}]^{+}, 471.0[\mathrm{M}-\mathrm{H}]^{-}$; ACPI-MS: $473.0[\mathrm{M}+\mathrm{H}]^{+}, 471.0[\mathrm{M}-\mathrm{H}]^{-}$. HRESI-MS found $[\mathrm{M}-\mathrm{H}]^{-}$ 471.0353; $\mathrm{C}_{25} \mathrm{H}_{11} \mathrm{O}_{10}[\mathrm{M}-\mathrm{H}]^{-}$requires 471.0352. IR (solid, $\mathrm{cm}^{-1}$ ): 3548, 3318, 3058, 1720, 1707, 1655, 1595, 1508, 1459, 1366, 1310, 1164, 1074, 1063, 1049, 983, 964, 793, 711, 672. ${ }^{1} \mathrm{H}$ NMR $\left(400 \mathrm{MHz}\right.$, DMSO- $\left.d_{6}\right) \delta: 8.13(1 \mathrm{H}, \mathrm{m}, \mathrm{H}-9), 7.90-$ 7.91 (1H, m, H-6), 7.84-7.86 (2H, m, H-7, 8), 7.36 (1H, s, H-15), $6.29(1 \mathrm{H}, \mathrm{s}, \mathrm{H}-22), 6.24(1 \mathrm{H}, \mathrm{s}, \mathrm{H}-24) .{ }^{13} \mathrm{C}$ NMR and DEPT $(101$ MHz, DMSO- $\left.d_{6}\right) \delta: 180.2$ (C-4), 177.0 (C-19), 175.0 (C-1), 164.3 (C23), 161.5 (C-21), 157.3 (C-25), 154.1 (C-2), 148.8 (C-14), 147.0 (C17), 146.8 (C-12), 136.9 (C-18), 134.8 (C-7), 134.4 (C-8), 133.6 (C10), 133.2 (C-13), 132.1 (C-5), 127.0 (C-6), 126.4 (C-9), 125.1 (C-3), 118.6 (C-15), 116.4 (C-16), 115.1 (C-11), 104.4 (C-20), 98.6 (C-22), 93.9 (C-24). ${ }^{1} \mathrm{H}$ was assigned to ${ }^{13} \mathrm{C}$ by HSQC correlations. In the ${ }^{1} \mathrm{H}^{1}{ }^{1} \mathrm{H}$ COSY spectrum, the couplings of $\mathrm{H}-6$ with $\mathrm{H}-7, \mathrm{H}-7$ with $\mathrm{H}-8, \mathrm{H}-8$ with $\mathrm{H}-9$ were determined. In the $\mathrm{HMBC}$ spectrum, the correlations of $\mathrm{H}-6$ with $\mathrm{C}-10 ; \mathrm{H}-7$ with $\mathrm{C}-9 ; \mathrm{H}-8$ with C-6; H-9 with $\mathrm{C}-1 ; \mathrm{H}-15$ with $\mathrm{C}-11,13,14 ; \mathrm{H}-22$ with $\mathrm{C}-20 ; \mathrm{H}-24$ with $\mathrm{C}-$ 20, 23, 25 were observed.

2: red powder; $126 \mathrm{mg}$, yield $18 \%$ (final product/myricetin $\times$ 100\%); mp 319-320 ${ }^{\circ} \mathrm{C}$. ESI-MS: $659.0\left[\mathrm{M}+\mathrm{CH}_{3} \mathrm{OH}-\mathrm{H}_{2} \mathrm{O}+\mathrm{H}\right]^{+}$, $657.0\left[\mathrm{M}+\mathrm{CH}_{3} \mathrm{OH}-\mathrm{H}_{2} \mathrm{O}-\mathrm{H}\right]^{-}$; ACPI-MS: $659.0\left[\mathrm{M}+\mathrm{CH}_{3} \mathrm{OH}-\mathrm{H}_{2} \mathrm{O}\right.$ $+\mathrm{H}]^{+}, 657.0\left[\mathrm{M}+\mathrm{CH}_{3} \mathrm{OH}-\mathrm{H}_{2} \mathrm{O}-\mathrm{H}\right]^{-}$. HRESI-MS found $[\mathrm{M}-\mathrm{H}]^{+}$ 643.0463; $\mathrm{C}_{35} \mathrm{H}_{16} \mathrm{O}_{13}[\mathrm{M}-\mathrm{H}]^{+}$requires 643.0518. IR (solid, $\mathrm{cm}^{-1}$ ): 3530, 3078, 1671, 1655, 1627, 1606, 1588, 1498,
1383, 1363, 1322, 1286, 1246, 1186, 1161, 1112, 1051, 975, 897, 841, 794, 711, 655. ${ }^{1} \mathrm{H}$ NMR (400 MHz, DMSO- $\left.d_{6}\right) \delta: 8.10-$ 8.12(1H, m, H-9), 7.99-8.01 (2H, m, H-31, 34), 7.94-7.96 (2H, m, $\mathrm{H}-32,33), 7.92-7.94$ (1H, m, H-6), 7.84-7.86 (2H, m, H-7, 8), 7.29 (1H, s, H-15), $6.40(1 \mathrm{H}, \mathrm{s}, \mathrm{H}-24), 6.20(1 \mathrm{H}, \mathrm{s}, \mathrm{H}-22) .{ }^{13} \mathrm{C}$ NMR and DEPT (101 MHz, DMSO- $\left.d_{6}\right) \delta: 182.3(\mathrm{C}-4), 177.4$ (C-29), 176.9 (C19), 176.6 (C-26), 174.9 (C-1), 165.0 (C-23), 162.0 (C-21), 160.1 (C27), 156.9 (C-25), 153.5 (C-14), 148.3 (C-17), 140.6 (C-2), 140.2 (C12), 139.5 (C-28), 138.5 (C-18), 134.9 (C-7, 32, 33), 134.7 (C-8), 132.7 (C-10), 132.2 (C-13), 131.1 (C-5), 130.6 (C-16), 130.2 (C35), 130.0 (C-30), 127.0 (C-3), 126.9 (C-31), 126.5 (C-9), 126.3 (C-34), 126.2 (C-6), 113.3 (C-15), 110.7 (C-11), 105.1 (C-20), 99.5 (C-22), 94.4 (C-24). ${ }^{1} \mathrm{H}$ was assigned to ${ }^{13} \mathrm{C}$ by HSQC correlations. In the ${ }^{1} \mathrm{H}^{-}{ }^{1} \mathrm{H}$ COSY spectrum, the couplings of $\mathrm{H}-6$ with $\mathrm{H}-7, \mathrm{H}-$ 7 with $\mathrm{H}-8, \mathrm{H}-8$ with $\mathrm{H}-9, \mathrm{H}-31$ with $\mathrm{H}-32, \mathrm{H}-32$ with $\mathrm{H}-33, \mathrm{H}-33$ with $\mathrm{H}-34$ were determined. In the $\mathrm{HMBC}$ spectrum, the correlations of $\mathrm{H}-6$ with $\mathrm{C}-7,8$; $\mathrm{H}-9$ with $\mathrm{C}-7,8$; $\mathrm{H}-15$ with $\mathrm{C}-11$, $13,14,17 ; \mathrm{H}-22$ with C-20, 24; H-31 with C-33; H-34 with C-32 were observed.

3: black powder; $112 \mathrm{mg}$, yield 16\% (final product/myricetin $\times 100 \%$ ); mp $425-426{ }^{\circ} \mathrm{C}$. ESI-MS: $659.0\left[\mathrm{M}+\mathrm{CH}_{3} \mathrm{OH}-\mathrm{H}_{2} \mathrm{O}+\right.$ $\mathrm{H}]^{+}, 656.8\left[\mathrm{M}+\mathrm{CH}_{3} \mathrm{OH}-\mathrm{H}_{2} \mathrm{O}-\mathrm{H}\right]^{-}$; ACPI-MS: $657.0\left[\mathrm{M}+\mathrm{CH}_{3} \mathrm{OH}-\right.$ $\left.\mathrm{H}_{2} \mathrm{O}-\mathrm{H}\right]^{-}$. HRESI-MS found $[\mathrm{M}-\mathrm{H}]^{+} 643.0463 ; \mathrm{C}_{35} \mathrm{H}_{16} \mathrm{O}_{13}[\mathrm{M}-$ $\mathrm{H}]^{+}$requires 643.0518. IR (solid, $\mathrm{cm}^{-1}$ ): 3647, 3317, 3071, 1673, 1645, 1630, 1603, 1591, 1571, 1508, 1474, 1434, 1369, 1320, 1277, 1246, 1226, 1211, 1178, 1156, 1104, 1060, 1010, 974, 948, 916, 900, 887, 833, 789, 750, 710, 695. ${ }^{1} \mathrm{H}$ NMR (400 MHz, DMSO- $\left.d_{6}\right) \delta: 8.09-8.11(1 \mathrm{H}, \mathrm{m}, \mathrm{H}-9), 8.04-8.05(1 \mathrm{H}, \mathrm{m}, \mathrm{H}-34)$, 7.99-8.01 (1H, m, H-31), 7.89-7.90 (1H, m, H-6), 7.87-7.88 (2H, m, H-32, 33), 7.82-7.86 (2H, m, H-7, 8), 7.56 (1H, s, H-15), $6.22(2 \mathrm{H}, \mathrm{s}, \mathrm{H}-22,24) .{ }^{13} \mathrm{C}$ NMR and DEPT $\left(101 \mathrm{MHz}, \mathrm{DMSO}-d_{6}\right)$ $\delta: 179.4$ (C-4), 177.0 (C-29), 177.0 (C-19), 176.8 (C-26), 174.9 (C1), 164.5 (C-23), 161.5 (C-21), 157.3 (C-25), 156.2 (C-27), 146.2 (C-17), 143.3 (C-2), 140.6 (C-14), 139.6 (C-28), 138.9 (C-12), 137.7 (C-18), 135.1-135.2 (C-7, 32, 33), 134.6 (C-8), 133.5 (C-10), 131.8 (C-5), 130.0 (C-35), 129.9 (C-30), 128.5 (C-13), 127.2 (C-6), 126.6 (C-9), 126.4 (C-31, 34), 124.0 (C-3), 123.0 (C-16), 121.1 (C-11), 117.8 (C-15), 104.6 (C-20), 98.8 (C-22), $94.0(\mathrm{C}-24) .{ }^{1} \mathrm{H}$ was assigned to ${ }^{13} \mathrm{C}$ by HSQC correlations. In the ${ }^{1} \mathrm{H}^{-1} \mathrm{H}$ COSY spectrum, the couplings of $\mathrm{H}-6$ with $\mathrm{H}-7, \mathrm{H}-7$ with $\mathrm{H}-8, \mathrm{H}-8$ with $\mathrm{H}-9, \mathrm{H}-31$ with $\mathrm{H}-32, \mathrm{H}-32$ with $\mathrm{H}-33, \mathrm{H}-33$ with $\mathrm{H}-34$ were determined. In the HMBC spectrum, the correlations of $\mathrm{H}-6$ with C-5; H-7 with C-9; H-8 with C-10; H-9 with C-7; $\mathrm{H}-15$ with C-11, 13, 14, 17; H-22 with C-20, 21, 23, 24; H-24 with C-20, 22, 23, 25; H-31 with C-33; H-32 with C-33, 34; H-33 with C-31, 32; H34 with C-32 were observed.

4: red columnar crystals. $133 \mathrm{mg}$, yield $20 \%$ (final product/ quercetin $\times 100 \%$ ); mp $263{ }^{\circ} \mathrm{C}$. ESI-MS: $263.0[\mathrm{M}-\mathrm{H}]^{-}$; ACPIMS: $265.0[\mathrm{M}+\mathrm{H}]^{+}, 263.0[\mathrm{M}-1]^{-}$. HRESI-MS found $[\mathrm{M}+\mathrm{H}]^{+}$ 265.0492; $\mathrm{C}_{16} \mathrm{H}_{9} \mathrm{O}_{4}[\mathrm{M}+\mathrm{H}]^{+}$requires 265.0495. Crystal structure, IR and NMR data were reported in our previous work. ${ }^{17}$

5: orange powder; $319 \mathrm{mg}$, yield $48 \%$ (final product/ quercetin $\times 100 \%$ ); mp $387-388{ }^{\circ} \mathrm{C}$. ESI-MS: $457.0[\mathrm{M}+\mathrm{H}]^{+}$, $455.0[\mathrm{M}-\mathrm{H}]^{-}$; ACPI-MS: $457.0[\mathrm{M}+\mathrm{H}]^{+}$, $455.0[\mathrm{M}-\mathrm{H}]^{-}$. HRESI-MS found $[\mathrm{M}+\mathrm{H}]^{+} 457.0554 ; \mathrm{C}_{25} \mathrm{H}_{13} \mathrm{O}_{9}[\mathrm{M}+\mathrm{H}]^{+}$requires 457.0554. IR (solid, $\mathrm{cm}^{-1}$ ): 3545, 3531, 3288, 3095, 1672, 1646, $1625,1600,1577,1559,1497,1438,1358,1305,1278,1218$, 
1193, 1166, 1090, 1015, 971, 943, 900, 842, 825, 798, 751, 709, 695. ${ }^{1} \mathrm{H}$ NMR (400 MHz, DMSO- $d_{6}$ ) $\delta: 8.12-8.14$ (1H, m, H-9), 7.91-7.93 (1H, m, H-6), 7.83-7.87 (1H, m, H-8), 7.76-7.80 $(1 \mathrm{H}$, $\mathrm{m}, \mathrm{H}-7), 7.61-7.63(1 \mathrm{H}, \mathrm{d}, J=8.3 \mathrm{~Hz}, \mathrm{H}-15), 7.22-7.24(1 \mathrm{H}, \mathrm{d}, J=$ $8.3 \mathrm{~Hz}, \mathrm{H}-14), 6.25(1 \mathrm{H}, \mathrm{d}, J=2.0 \mathrm{~Hz}, \mathrm{H}-22), 6.22(1 \mathrm{H}, \mathrm{d}, J=$ $2.0 \mathrm{~Hz}, \mathrm{H}-24) .{ }^{13} \mathrm{C}$ NMR and DEPT (101 MHz, DMSO- $\left.d_{6}\right) \delta: 179.9$ (C-4), 177.1 (C-19), 175.5 (C-1), 164.3 (C-23), 161.5 (C-21), 157.3 (C-25), 154.7 (C-2), 149.1 (C-17), 145.9 (C-13), 145.4 (C-12), 137.0 (C-18), 135.0 (C-7), 134.4 (C-8), 133.7 (C-10), 132.1 (C-5), 129.8 (C-15), 127.1 (C-6), 126.5 (C-9), 124.5 (C-3), 123.3 (C-11), 116.9 (C-16), 115.0 (C-14), 104.5 (C-20), 98.6 (C-22), 93.9 (C-24). ${ }^{1} \mathrm{H}$ was assigned to ${ }^{13} \mathrm{C}$ by HSQC correlations. In the ${ }^{1} \mathrm{H}-{ }^{1} \mathrm{H}$ COSY spectrum, the couplings of $\mathrm{H}-6$ with $\mathrm{H}-7, \mathrm{H}-7$ with $\mathrm{H}-8, \mathrm{H}-8$ with $\mathrm{H}-9$ were determined. In the $\mathrm{HMBC}$ spectrum, the correlations of H-6 with C-8; H-8 with C-5, 6, 9, 10; H-9 with C-7; H-14 with C12, 16; H-15 with C-11, 13, 17; H-22 with C-20, 21, 24; H-24 with C-20, 22, 25 were observed.

6: brownish red powder; $98 \mathrm{mg}$, yield 15\% (final product/ quercetin $\times 100 \%$ ); mp 390-391 ${ }^{\circ} \mathrm{C}$. ESI-MS: $629.0[\mathrm{M}+\mathrm{H}]^{+}$, $627.0[\mathrm{M}-\mathrm{H}]^{-}$; ACPI-MS: $629.0[\mathrm{M}+\mathrm{H}]^{+}$. HRESI-MS found $[\mathrm{M}+$ $\mathrm{H}]^{+}$629.0711; $\mathrm{C}_{35} \mathrm{H}_{17} \mathrm{O}_{12}[\mathrm{M}+\mathrm{H}]^{+}$requires 629.0715. IR (solid, $\mathrm{cm}^{-1}$ ): 3651, 3292, 3125, 2925, 2851, 1654, 1636, 1590, 1560, 1508, 1474, 1368, 1306, 1264, 1215, 1192, 1165, 1090, 1073, 1011, 981, 941, 899, 796, 711, 696. ${ }^{1} \mathrm{H}$ NMR $(400 \mathrm{MHz}$, DMSO- $\left.d_{6}\right) \delta:$ 8.15-8.18 (1H, m, H-9), 8.09-8.11 (1H, m, H-34), 7.99-8.02 (1H, m, H-31), 7.95-7.97 (1H, m, H-6), 7.89-7.91 (1H, m, H-8), 7.88-7.89 (2H, m, H-32, 33), 7.85-7.87 (1H, m, H7), 7.62-7.64 (1H, d, $J=8.4 \mathrm{~Hz}, \mathrm{H}-15), 7.48-7.50(1 \mathrm{H}, \mathrm{d}, J=$ $8.4 \mathrm{~Hz}, \mathrm{H}-14), 6.26-6.27(1 \mathrm{H}, \mathrm{d}, J=2.0 \mathrm{~Hz}, \mathrm{H}-22), 6.23-6.24(1 \mathrm{H}$, $\mathrm{d}, J=2.0 \mathrm{~Hz}, \mathrm{H}-24) .{ }^{13} \mathrm{C}$ NMR and DEPT (101 MHz, DMSO- $\left.d_{6}\right) \delta$ : 182.0 (C-29), 181.5 (C-26), 179.8 (C-4), 177.1 (C-19), 175.4 (C-1), 164.4 (C-23), 161.5 (C-21), 157.4 (C-25), 155.2 (C-2), 148.4 (C17), 144.8 (C-13), 144.8 (C-12), 137.3 (C-18), 135.8 (C-28), 135.1 (C-7), 135.1 (C-33), 134.7 (C-27), 134.5 (C-8), 134.1 (C-32), 133.7 (C-10), 132.2 (C-5), 131.3 (C-30), 130.9 (C-35), 129.4 (C-15), 127.2 (C-6), 126.6 (C-9), 126.5 (C-34), 126.2 (C-31), 124.2 (C-3), 123.7 (C-11), 120.3 (C-16), 114.3 (C-14), 104.5 (C-20), 98.7(C-22), 94.0 (C-24). ${ }^{1} \mathrm{H}$ was assigned to ${ }^{13} \mathrm{C}$ by HSQC correlations. In the ${ }^{1} \mathrm{H}-{ }^{1} \mathrm{H}$ COSY spectrum, the couplings of $\mathrm{H}-6$ with $\mathrm{H}-7, \mathrm{H}-7$ with $\mathrm{H}-8, \mathrm{H}-8$ with $\mathrm{H}-9, \mathrm{H}-14$ with $\mathrm{H}-15, \mathrm{H}-31$ with $\mathrm{H}-32$, $\mathrm{H}-32$ with $\mathrm{H}-$ 33, $\mathrm{H}-33$ with $\mathrm{H}-34$ were determined.

7: red needle crystals; $326 \mathrm{mg}$, yield $66 \%$ (final product/ chalcone $\times 100 \%) ; \mathrm{mp} 264-265{ }^{\circ} \mathrm{C}$. Its crystal structure, MS, IR and NMR data were reported in our previous work. ${ }^{18}$

8: red needle crystals; $336 \mathrm{mg}$, yield $68 \%$ (final product/ chalcone $\times 100 \%)$; mp 258-259 ${ }^{\circ} \mathrm{C}$. ESI-MS: $352.2[\mathrm{M}+\mathrm{H}]^{+}$; ACPI-MS: $352.2[\mathrm{M}+\mathrm{H}]^{+}$. HRESI-MS found $[\mathrm{M}+\mathrm{Na}]^{+}$374.0792, $[2 \mathrm{M}+\mathrm{Na}]^{+} 725.1689 ; \mathrm{C}_{23} \mathrm{H}_{13} \mathrm{NO}_{3} \mathrm{Na}[\mathrm{M}+\mathrm{Na}]^{+}$requires 374.0788, $\mathrm{C}_{46} \mathrm{H}_{26} \mathrm{~N}_{2} \mathrm{O}_{6} \mathrm{Na}[2 \mathrm{M}+\mathrm{Na}]^{+}$requires 725.1683. IR (solid, $\mathrm{cm}^{-1}$ ): 3690, 3650, 3110, 3098, 3079, 3056, 2956, 2919, 1851, 1667, 1640, 1623, 1588, 1570, 1490, 1466, 1447, 1392, 1365, 1307, 1276, 1227, 1209, 1159, 1134, 1062, 1037, 1018, 998, 905, 877, 834, 808, 758, 748, 706, 693, 662. ${ }^{1} \mathrm{H}$ NMR (500 MHz, $\left.\mathrm{CDCl}_{3}\right) \delta$ : 9.83-9.85 (1H, d, $J=7.0 \mathrm{~Hz}, \mathrm{H}-11), 8.27-8.29(1 \mathrm{H}, \mathrm{d}, J=7.5 \mathrm{~Hz}$, H-6), 8.03-8.05 (1H, d, $J=7.5 \mathrm{~Hz}, \mathrm{H}-9), 7.97-7.99$ (1H, d, $J=$ $9.0 \mathrm{~Hz}, \mathrm{H}-14), 7.91-7.93$ (2H, d, $J=7.5 \mathrm{~Hz}, \mathrm{H}-19,23), 7.74-7.77$ $(1 \mathrm{H}, \mathrm{td}, J=7.5,1.1 \mathrm{~Hz}, \mathrm{H}-7), 7.65-7.68\left(1 \mathrm{H}, \mathrm{td}, J=7.5,1.1 \mathrm{~Hz}, \mathrm{H}^{-}\right.$
8), 7.60-7.63 (1H, t, $J=7.5 \mathrm{~Hz}, \mathrm{H}-21), 7.46-7.49(2 \mathrm{H}, \mathrm{t}, J=$ $7.5 \mathrm{~Hz}, \mathrm{H}-20,22), 7.41-7.44$ (1H, m, H-13), 7.21-7.24 (1H, t, $J=$ $7.3 \mathrm{~Hz}, \mathrm{H}-12) .{ }^{13} \mathrm{C}$ NMR and DEPT (126 MHz, $\mathrm{CDCl}_{3}$ ) $\delta: 191.9$ (C17), 180.9 (C-1), 175.0 (C-4), 139.2(C-15), 138.9(C-18), 134.3(C-5), 133.8(C-7), 133.6 (C-10), 133.1 (C-8), 133.1 (C-21), 129.3 (C-19, 23), 128.4 (C-20, 22), 128.4 (C-3), 128.3 (C-13), 127.8 (C-11), 127.2 (C-9), 126.4 (C-6), 121.4 (C-2), 120.4 (C-14), 117.8 (C-12), 113.7 (C-16). ${ }^{1} \mathrm{H}$ was assigned to ${ }^{13} \mathrm{C}$ by HSQC correlations. In the ${ }^{1} \mathrm{H}-{ }^{1} \mathrm{H}$ COSY spectrum, the couplings of $\mathrm{H}-6$ with $\mathrm{H}-7, \mathrm{H}-7$ with $\mathrm{H}-8, \mathrm{H}-8$ with $\mathrm{H}-9, \mathrm{H}-11$ with $\mathrm{H}-12, \mathrm{H}-12$ with $\mathrm{H}-13, \mathrm{H}-13$ with $\mathrm{H}-14, \mathrm{H}-19$ with $\mathrm{H}-20, \mathrm{H}-20$ with $\mathrm{H}-21, \mathrm{H}-21$ with $\mathrm{H}-22$, $\mathrm{H}-22$ with $\mathrm{H}-23$ were determined. In the HMBC spectrum, the correlations of $\mathrm{H}-6$ with C-4, 8, 10; H-7 with C-5, 9; H-8 with C-6, 7, 10; H-9 with C-1, 5, 7; H-11 with C-3, 12, 13, 15; H-12 with C13 , 14; H-13 with C-11, 12, 15; H-14 with C-12, 15; H-19 with C17, 21, 23; H-20 with C-18, 19, 21, 22; H-21 with C-19, 23; H-22 with C-18, 20, 21, 23; H-23 with C-17, 19, 21 were observed.

9: red needle crystals; $389 \mathrm{mg}$, yield $65 \%$ (final product/ naringenin $\times 100 \%$ ); mp $318-319{ }^{\circ} \mathrm{C}$. ESI-MS: $368.0[\mathrm{M}+\mathrm{H}]^{+}$, 366.0 $[\mathrm{M}-\mathrm{H}]^{-}$; ACPI-MS: $368.0[\mathrm{M}+\mathrm{H}]^{+}$, 366.0 $[\mathrm{M}-\mathrm{H}]^{-}$. HRESI-MS found $[\mathrm{M}+\mathrm{H}]^{+} 368.0916 ; \mathrm{C}_{23} \mathrm{H}_{14} \mathrm{NO}_{4}[\mathrm{M}+\mathrm{H}]^{+}$ requires 368.0917. IR (solid, $\mathrm{cm}^{-1}$ ): 3650, 3358, 3071, 1670, 1629, 1614, 1599, 1566, 1560, 1491, 1474, 1436, 1394, 1368, 1306, 1276, 1226, 1211, 1162, 1137, 1063, 1037, 906, 878, 845, $777,755,713,704,688 .{ }^{1} \mathrm{H}$ NMR (400 MHz, DMSO- $\left.d_{6}\right) \delta: 10.41$ (1H, s, 21-OH), 9.63-9.66 (1H, m, H-11), 8.12-8.15 (1H, m, H-6), 7.91-7.93 (1H, m, H-9), 7.80-7.85 (1H, m, H-7), 7.73-7.77 (4H, $\mathrm{m}, \mathrm{H}-8,14,19,23), 7.47-7.51(1 \mathrm{H}, \mathrm{m}, \mathrm{H}-13), 7.36-7.39$ (1H, m, H12), 6.80-6.82 (2H, d, $J=8.5 \mathrm{~Hz}, \mathrm{H}-20,22) .{ }^{13} \mathrm{C}$ NMR and DEPT (101 MHz, DMSO- $d_{6}$ ) $\delta: 189.5$ (C-17), 181.1 (C-1), 174.1 (C-4), 162.8 (C-21), 138.1(C-15), 134.6 (C-7), 134.6 (C-5), 133.7 (C-8), 133.5 (C-10), 132.4 (C-19, 23), 130.1 (C-18), 128.3 (C-13), 128.1 (C-11), 127.7 (C-3), 126.9 (C-9), 126.4 (C-6), 120.9 (C-2), 120.1 (C14), 118.7 (C-12), 115.7 (C-20, 22), 114.3 (C-16). ${ }^{1} \mathrm{H}$ was assigned to ${ }^{13} \mathrm{C}$ by HSQC correlations. In the ${ }^{1} \mathrm{H}-{ }^{1} \mathrm{H}$ COSY spectrum, the couplings of $\mathrm{H}-6$ with $\mathrm{H}-7, \mathrm{H}-7$ with $\mathrm{H}-8, \mathrm{H}-8$ with $\mathrm{H}-9, \mathrm{H}-11$ with $\mathrm{H}-12$, H-12 with $\mathrm{H}-13$, H-13 with $\mathrm{H}-14, \mathrm{H}-19$ with $\mathrm{H}-20, \mathrm{H}-22$ with $\mathrm{H}-23$ were determined. In the $\mathrm{HMBC}$ spectrum, the correlations of H-6 with C-4, 10; H-7 with C-5, 9; H-8 with C-6, 10; H-9 with C-1, 5, 7; H-11 with C-3, 12, 13, 15; H-12 with C11, 13, 14; H-13 with C-11, 15; H-14 with C-12, 15; H-19 with C-17, 21; H-20 with C-18, 21, 22; H-22 with C-18, 20, 21; H-23 with $\mathrm{C}-17,21 ; 21-\mathrm{OH}$ with $20,21,22$ were observed.

10: yellow needle crystals; 300-599 mg, yield 20-40\% (final product/dichlone $\times 100 \%)$; mp 296-297 ${ }^{\circ} \mathrm{C}$. ESI-MS: $252.2[\mathrm{M}+$ $\mathrm{H}]^{+}$; ACPI-MS: $252.2[\mathrm{M}+\mathrm{H}]^{+}$. HRESI-MS found $[\mathrm{M}+\mathrm{H}]^{+}$ 252.0662; $\mathrm{C}_{15} \mathrm{H}_{10} \mathrm{NO}_{3}[\mathrm{M}+\mathrm{H}]^{+}$requires 252.0655. ${ }^{1} \mathrm{H} \mathrm{NMR}(400$ MHz, DMSO- $\left.d_{6}\right) \delta: 8.89-8.91(2 \mathrm{H}, \mathrm{d}, J=5.7 \mathrm{~Hz}, \mathrm{H}-11,15), 8.57-$ $8.61(1 \mathrm{H}, \mathrm{t}, J=7.6 \mathrm{~Hz}, \mathrm{H}-13), 8.15-8.19(2 \mathrm{H}, \mathrm{t}, J=6.7 \mathrm{~Hz}, \mathrm{H}-12$, 14), 8.05-8.07 (1H, d, $J=7.5 \mathrm{~Hz}, \mathrm{H}-6), 7.96-7.98(1 \mathrm{H}, \mathrm{d}, J=$ $7.5 \mathrm{~Hz}, \mathrm{H}-9), 7.81-7.85(1 \mathrm{H}, \mathrm{t}, J=7.4 \mathrm{~Hz}, \mathrm{H}-7), 7.70-7.73(1 \mathrm{H}, \mathrm{t}, J$ $=7.3 \mathrm{~Hz}, \mathrm{H}-8) .{ }^{13} \mathrm{C}$ NMR and DEPT $\left(101 \mathrm{MHz}\right.$, DMSO- $\left.d_{6}\right) \delta: 184.4$ (C-1), 172.4 (C-4), 165.4 (C-3), 148.1 (C-11,15), 145.1 (C-13), 134.9 (C-7), 134.1 (C-5), 132.1 (C-8), 131.6 (C-10), 127.4 (C-12, 14), 126.5 (C-9), 126.3 (C-6), 125.4 (C-2). ${ }^{1} \mathrm{H}$ was assigned to $13 \mathrm{C}$ by HSQC correlations. In the ${ }^{1} \mathrm{H}-{ }^{1} \mathrm{H}$ COSY spectrum, the couplings of $\mathrm{H}-6$ with $\mathrm{H}-7$; H-7 with $\mathrm{H}-8$; H-8 with $\mathrm{H}-9$; $\mathrm{H}-13$ with $\mathrm{H}-12$, 14; 
$\mathrm{H}-11$ with $\mathrm{H}-12 ; \mathrm{H}-14$ with $\mathrm{H}-15$ were determined. In the $\mathrm{HMBC}$ spectrum, the correlations of $\mathrm{H}-6$ with $\mathrm{C}-4,10$; $\mathrm{H}-7$ with $\mathrm{C}-5,9$; H-8 with C-6, 10; H-9 with C-1, 5; H-11, 15 with C-13; H-13 with C-11, 15; H-12 with C-11; H-14 with C-15; H-12 with C-14; $\mathrm{H}-14$ with $\mathrm{C}-12$ were observed.

\subsection{Synthesis and isolation}

One of the selected flavonoids $(0.002 \mathrm{~mol})$ was mixed with dichlone $(0.006 \mathrm{~mol})$ uniformly, and then $50 \mathrm{~mL}$ of pyridine was added to dissolve the mixed solid in a 100-mL double mouth round bottom flask. The reacting system was stirred at $85^{\circ} \mathrm{C}$ for $24 \mathrm{~h}$ (in order to synthesize 4, 7, 8, 9, additional conditions, air being pumped through a water-filled absorption bottle and then injected into this mixture solution with flow velocity of 1 bubble/2 s, must be provided), and was finally concentrated to give a semi-solid mass. The mass was chromatographed on Biotage Isolera Four flash column chromatography system with a SNAP Cartridge KP-Sil $10 \mathrm{~g}$ column eluted by hexane/ethyl acetate $(100: 0$ to $90: 10, \mathrm{v} / \mathrm{v})$, and then by chloroform/ methanol $(100: 0$ to $90: 10, \mathrm{v} / \mathrm{v})$ to give fractions. TLC was used to distinguish the eluted fractions. The fractions were further isolated by preparative TLC (1000 microns; $20 \mathrm{~cm} \times 20$ $\mathrm{cm}$ ) using chloroform/methanol or hexane/ethyl acetate as developing agent to yield derivatives.

\subsection{Assay for anticancer activity}

Cytotoxicity against non-solid tumor and solid tumor were tested by Trypan blue dye exclusion method and Neutral red uptake method, respectively. ${ }^{21} \mathrm{IC}_{50}$ was calculated from the dose curves generated by plotting percent growth $v s$. the test concentration on a logarithmic scale using Microsoft Excel. All assays were performed in triplicate and the mean values are given in Table 1.

\subsection{Apoptosis analysis}

The sensitive cancer cell lines were separately incubated with derivatives, DMSO and doxorubicin (positive control) at different concentrations for $24 \mathrm{~h}$, harvested by centrifugation at $300 \mathrm{~g}$, washed twice with cold $1 \times$ PBS and resuspended in $100 \mu \mathrm{L}$ of binding buffer at a density of $2 \times 10^{6}$ cells per $\mathrm{mL}$, stained with $5 \mu \mathrm{L}$ of PE Annexin-V and $5 \mu \mathrm{L}$ of 7-AAD for $15 \mathrm{~min}$ in the dark at room temperature and finally subjected to analysis via Accuri $^{\text {TM }}$ C6 flow cytometry. ${ }^{22,23}$

\subsection{Western blotting analysis}

The sensitive cancer cell lines were separately treated with derivatives, doxorubicin (positive control) and DMSO at different concentrations for $24 \mathrm{~h}$, washed with cold $1 \times$ PBS, harvested in RIPA lysis solution for $60 \mathrm{~min}$ on ice, and then heated to boiling point for 10 min after adding loading buffer. The cell lysate were centrifuged at $13000 \mathrm{~g}$ for $10 \mathrm{~min}$ at $4{ }^{\circ} \mathrm{C}$. Equal amounts of the protein samples were subjected to SDSPAGE electrophoresis and transferred to PVDF membranes. The membranes were blocked in $5 \%$ nonfat milk, incubated with diluted primary antibodies and secondary antibodies conjugated with HRP, treated with Western Lighting Chemiluminescence Reagent and finally visualized on biomolecular imager to determine the expression levels of apoptosis marker proteins including caspase- 3 and PARP cleavage (using $\beta$-actin as internal reference). ${ }^{23,24}$

\section{Conclusion}

By the common base-catalyzed method, not only mono condensation products $(\mathbf{1}, \mathbf{5})$ but also bis-naphthoquinones compounds $(2,3,6)$ were obtained.

Through a new method of combining base-catalyzed with $\mathrm{O}_{2} /$ $\mathrm{H}_{2} \mathrm{O}$ exposing, benzonaphthofuroquinone (4) and benzoylnaphthindolizinediones $(\mathbf{7}, \mathbf{8}, \mathbf{9})$ were synthesized. To our knowledge this is the first report about the reaction of chalcone/ dihydroflavone with dichlone under given conditions to produce benzoylnaphthindolizinediones, as well as the reaction of flavonols with dichlone to achieve benzonaphthofuroquinones. Benzoylnaphthindolizinediones were previously synthesized by methods of potassium carbonate-catalyzing, ${ }^{11}$ or copper(II)-catalyzing, ${ }^{12}$ or iodine oxidation. ${ }^{13}$

Some of the derivatives $(2,3,4,5)$ showed good anticancer activities and low toxicity to normal cells, which indicated that they possess some cytotoxic selectivity. The formed furan-ring in benzonaphthofuroquinones being replaced by pyrrol-ring in benzoylnaphthindolizinediones resulted in the loss of anticancer activity. Late-stage-apoptosis and caspase-3 pathway may contribute to the cytotoxicities of the synthesized benzonaphthofuroquinones.

\section{Conflicts of interest}

There are no conflicts to declare.

\section{Acknowledgements}

This work was funded by State Scholarship Fund awarded by China Scholarship Council, 201706660015; Guangxi Scholarship Fund of Guangxi Education Department, guijiaoshipei2016-22; Promotion of Young and Middle-aged Teachers' Basic Scientific Research Ability in Guangxi Universities by Guangxi Education Department, 2019KY0329; Opening Project of First-class Discipline Construction in Guangxi, 2019XK047, 2019XK132 and Guangxi Key Laboratory of Zhuang and Yao Ethnic Medicine, guikejizi-2014-32, GXZYZZ2019-7, GXZYKF2019-6.

\section{Notes and references}

1 E.-L. Bonifazi, C. Rios-Luci, L.-G. Leon, G. Burton, J.-M. Padron and R.-I. Misico, Bioorg. Med. Chem., 2010, 18, 2621-2630.

2 M.-G. Miller, A. Rodgers and G.-M. Cohen, Biochem. Pharmacol., 1986, 35, 1177-1184.

3 H. Sheridan, C. Nestor, L. O'Driscoll and I. Hook, J. Nat. Prod., 2011, 74, 82-85. 
4 A. Morello, M. Pavani, J.-A. Garbarino, M.-C. Chamy, C. Frey, J. Mancilla, A. Guerrero, Y. Repetto and J. Ferreira, Comp. Biochem. Physiol., Part C: Pharmacol., Toxicol. Endocrinol., 1995, 112, 119-128.

5 H. Cho, S.-J. Park and K.-I. Lee, Bull. Korean Chem. Soc., 2005, 26, 1829-1832.

6 K.-I. Lee, Y. Park, S.-J. Park, J.-H. Hwang, S.-J. Lee, G.-D. Kim, W.-K. Park, S. Lee, D. Jeong, J.-Y. Kong, H.-K. Kang and H. Cho, Bioorg. Med. Chem. Lett., 2006, 16, 737-742.

7 J. Padwal, W. Lewis and C.-J. Moody, J. Org. Chem., 2011, 76, 8082-8087.

8 C.-C. Cheng, Q. Dong, D.-F. Liu, Y.-L. Luo, L.-F. Liu, A.-Y. Chen, C. Yu, N. Savaraj and T.-C. Chou, J. Med. Chem., 1993, 36, 4108-4112.

9 H. Suginome, A. Konishi, H. Sakurai, H. Minakawa, T. Takeda, H. Senboku, M. Tokuda and K. Kobayashi, Tetrahedron, 1995, 51, 1377-1386.

10 T. Takeya, H. Kondo, T. Otsuka, K. Tomita, I. Okamoto and O. Tamura, Org. Lett., 2007, 9, 2807-2810.

11 Y. Liu, H.-Y. Hu, Q.-J. Liu, H.-W. Hu and J.-H. Xu, Tetrahedron, 2007, 63, 2024-2033.

12 Y. Liu and J.-W. Sun, J. Org. Chem., 2012, 77, 1191-1197.

13 A. Citterio, M. Fochi, A. Marion, A. Mele, R. Sebastiano and M. Delcanale, Heterocycles, 1998, 48, 1993-2002.

14 L.-Y. Xie, S. Peng, J.-X. Tan, R.-X. Sun, X.-Y. Yu, N.-N. Dai, Z.-L. Tang, X.-H. Xu and W.-M. He, ACS Sustainable Chem. Eng., 2018, 6, 16976-16981.
15 S. Peng, Y.-X. Song, J.-Y. He, S.-S. Tang, J.-X. Tan, Z. Cao, Y.-W. Lin and W.-M. He, Chin. Chem. Lett., 2019, 30, 22872290.

16 W.-H. Bao, M. He, J.-T. Wang, X. Peng, M. Sung, Z.-L. Tang, S. Jiang, Z. Cao and W.-M. He, J. Org. Chem., 2019, 84, 60656071.

17 P. Luo, A.-G. Chittiboyina, W.-G. Pan and W.-X. Wei, Z. Kristallogr. - New Cryst. Struct., 2020, 235, 565-567.

18 P. Luo, A.-G. Chittiboyina, M. Wang, I.-A. Khan, W.-G. Pan and W.-X. Wei, Z. Kristallogr. - New Cryst. Struct., 2020, 235, 105-107.

19 A. Schmidt and M. Albrecht, Z. Naturforsch., B: J. Chem. Sci., 2008, 63, 465-472.

20 J.-P. Guthrie, J. Cossar, P.-A. Cullimore, N.-M. Kamkar and K.-F. Taylor, Can. J. Chem., 1983, 61, 2621-2626.

21 J. Mustafa, S.-I. Khan, G.-Y. Ma, L.-A. Walker and I.-A. Khan, Lipids, 2004, 39, 167-172.

22 I. Vermes, C. Haanen, H. Steffens-Nakken and C. Reutelingsperger, J. Immunol. Methods, 1995, 184, 39-51.

23 J. Wu, Y. Ding, C.-H.-Z. Chen, Z.-M. Zhou, C.-Y. Ding, H.-Y. Chen, J. Zhou and C.-S. Chen, Cancer Lett., 2016, 380, 393-402.

24 Y.-J. Kong, F.-B. Li, Y. Nian, Z.-M. Zhou, R.-X. Yang, M.-H. Qiu and C.-S. Chen, Theranostics, 2016, 6, 875-886. 